\title{
Human Trajectory Prediction Model and its Coupling with a Walking Pattern Generator of a Humanoid Robot*
}

\author{
I. Maroger ${ }^{1}$, N. Ramuzat ${ }^{1,2}$, O. Stasse ${ }^{1}$ and B. Watier ${ }^{1}$
}

\begin{abstract}
In order to smoothly perform interactions between a humanoid robot and a human, knowledge about the human locomotion can be efficiently used. Indeed, in a human-robot collaboration, a prediction model of the human behaviour allows the robot to act proactively. In this paper, an optimal control based model predicting the human Center of Mass (CoM) trajectory during gait is presented. A Walking Pattern Generator (WPG) based on non-linear model predictive control is, then, introduced in order to generate the robot CoM and footsteps along the predicted trajectory. The combination of the human trajectory prediction model and this new WPG aims to allow the robot to proactively walk along with a human instead of passively follow him. These models have been tested in simulation on Gazebo on a TALOS humanoid robot model using measured human trajectories. To perform the CoM and foot trajectories computed by the WPG, a real-time whole-body controller is used. This controller is a Quadratic Program which solves the inverse dynamics of the robot at torque level.
\end{abstract}

Index Terms-humanoid robot, human locomotion, optimal control, prediction, walking pattern generator

\section{INTRODUCTION}

Interactions between human and humanoid robots raise great challenges. Indeed, not only are the human behaviours not always well known but also the humanoid robots are complex to control. Furthermore, the redundancy of the musculoskeletal system allows multiple behaviours which make them hard to predict. This paper is part of a French project, called ANR-COBOT, which aims at a collaboration between a human and a TALOS humanoid robot to carry and move a table. To smoothly achieve such a handling task, a good knowledge of the human dynamics while walking with a table is needed to allow the robot to anticipate and proactively follow the human movements. For now, the problem of walking with a table has been put aside and the issue has been reduced to the proactive tracking of a walking human by a humanoid robot. Thus, this work focuses on the real-time prediction of human walking trajectory and its coupling with a Walking Pattern Generator (WPG) for a TALOS humanoid robot.

\section{A. State of the art}

1) Proactive human-robot interactions: To proactively collaborate with a human, a humanoid robot needs to predict, or at least guess, its partner's future actions [1]. As far as we

* I. Maroger, B. Watier and O. Stasse are funded by the grant ANRCOBOT [18CE10-0003]. N. Ramuzat is funded by the research agreement ROB4FAM.

${ }^{1}$ LAAS-CNRS, Université de Toulouse, CNRS, UPS, Toulouse, France

${ }^{2}$ Airbus OMIR, Toulouse, France

\{imaroger, nramuzat, ostasse, bwatier\}@laas.fr

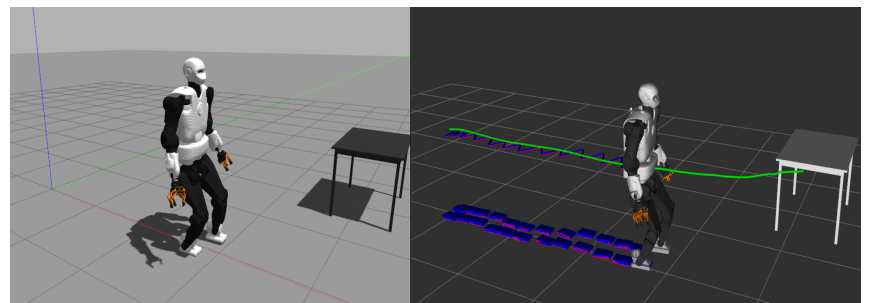

Fig. 1. Simulation on Gazebo (left) of the robot executing the predicted trajectory with $N_{0}=50$ and $N_{O C}=100$. The CoM, footsteps (desired in red, real in blue) and human trajectories (in green) are also displayed in RViz (right) for comparison.

know, the first experiment where a humanoid robot proactively interacts with a human was performed in [2]. In this work motion primitives like Stop, Side, Turn and Walk/Turn were used to generate the robot locomotion according to the human velocity. Since then, collaborative tasks are often aimed to be as proactive as possible in order to smooth the human-robot interactions.

For example, in [3], a co-manipulation task is aimed. To achieve this goal, the authors design a robot controller which can generate optimal motions in real time equivalent to those generated by a human. As this controller takes into account a whole-body dynamics of the human, it allows a more proactive interaction between the humanoid-robot and the human. In this paper, a similar method is enforced, only the goal differs. Indeed, here, we try to perform a co-navigation task instead of a co-manipulation task.

Furthermore, proactive co-navigation tasks have already been well studied. Indeed, in [4], the authors propose a reactive trajectory planner for robot which takes into account the human predicted motions and goals to handle humanrobot co-navigation. The estimation of the human movement is based on its current velocity and three different navigation modes are presented to make the robot move forward without colliding with the human. Thus, even if our work also aims a co-navigation task, the goal is not to smoothly avoid but to walk along with a human partner.

The co-navigation task we are trying to perform in this article is the first step toward a table handling task which can be compared to one aimed in [5] and [6]. Indeed, in those papers, the authors target a collaborative handling task while walking, where the humanoid robot identifies its human partner intentions. To guess human future motions, a training of a multiclass classifier of human intentions was performed using measurements collected from a human-human handling collaboration. The results of this training, namely the optimal features to discriminate a set of human motions, was then 


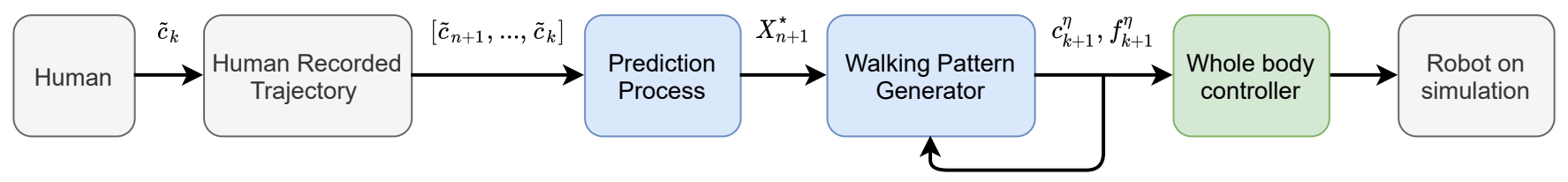

Fig. 2. Description of the whole framework presented in this paper

used to predict human intentions. In this article, instead of using supervised learning to classify and guess human intentions, an Optimal Control (OC) scheme is built to predict the human future trajectory. To the best of our knowledge, machine learning [7] [8] [9] and probabilistic state machines [10] have already been studied to guess human intentions but it's the first time that an OC model is proposed to solve such a real-time prediction problem. A real-time approach is more complex but gives more versatility for future works. Indeed, optimisation problems allow to incrementally add obstacles or new constraints by adding new terms to the cost function.

2) Human trajectories during gait: Healthy human gait is a well studied field in biomechanics [11]. However, as far as we can tell, the focus of those works is rarely on the modeling and the simulation of human walking trajectories. Nevertheless, in robotics, some authors studied this problem to generate human-like paths for a robot to follow. Thus, models of human walking trajectories using parametric curves called clothoids [12] or OC models [13] [14] [15] have been designed. In [16], the authors assessed those models according to a metrics evaluating the linear and angular distances between human trajectories and generated trajectories. The most human-like model was the one introduced in [15] which approximates the human by a holonomic system instead of a non-holonomic one. Indeed, holonomic model allows lateral and oblique motions which better fits human locomotion. This is why a new OC model, inspired from [15], is defined in [17]. This model gives an accurate approximation of human behaviour. We will use this OC model in the following of this paper.

3) Humanoid robot WPG: The generation of a stable humanoid robot gait is a commonly tackled issue in robotics. Introduced by [18], the Linear Inverted Pendulum Model (LIPM) is widely used to approximate the non linear dynamics of a humanoid robot Center of Mass (CoM) during gait as a function of the Zero Moment Point (ZMP). Indeed, the ZMP is a relevant variable to control as the ZMP has to stay within the support polygon of the robot at all time to ensure the humanoid robot balance [19].

In [20], the authors present a ZMP preview control which computes the CoM of the humanoid robot over a prediction horizon from imposed footsteps given to the controller. In [21], a new formulation of this ZMP preview control using a Model Predictive Control (MPC) scheme is proposed. Nowadays, this MPC approach is the most commonly adopted method to design a WPG for humanoid robots [22] [23] [24]. Moreover, the original MPC has been improved to achieve automatic footstep placements by [25]. Even non linear reformulations of this MPC were proposed in [26] [27]. The WPG presented in this work is based on the Nonlinear Model Predictive Control (NMPC) proposed by [26] which allows to simultaneously compute the CoM and the footsteps positions and orientations. Although only linear constraints are implemented in this paper, this NMPC scheme is able to deal with non-linear constraints to avoid obstacles which will be useful in future works to avoid collisions between the robot and the table feet for example.

\section{B. Contributions}

This article presents a prediction model of human trajectory during gait and its coupling with a WPG which generates a TALOS humanoid robot CoM and footsteps along the predicted trajectory. This coupling is aimed to allow a proactive tracking of a walking human by a humanoid robot, instead of a passive one, in order to ease every potential interactions during gait. The whole framework is assessed in simulation on Gazebo, as Fig 1 shows, and is described on Fig 2. The focus of this paper is on the two blue boxes which are presented in section II and III Their coupling and the green box are then presented in section IV.

The contributions of this paper are three-fold. First, a prediction OC model is designed based on a human trajectory model. This prediction model is expected to provide an accurate estimation of the human future trajectory. Then, a new WPG using NMPC* is developed to generate a TALOS robot CoM and footsteps along a given trajectory. Finally, the coupling of this WPG with the prediction model is simulated to assess the efficiency of this process. This framework is designed to help a humanoid robot to predict the spontaneous human trajectory during gait according to its real-time CoM observation. This aims to allow the robot to move in accordance with its human partner. Thus, if the human accelerates, slows down or stops, the robot should act similarly while following the same trajectory as its partner.

\section{PREDiction of HUMAN TRAJECtORIES DURING Gait}

First of all, to make the robot anticipate the human behaviour during gait, a model which allows to predict where a human is going using its past trajectory is needed. In this part, such a model, based on the OC model described in [17], is presented.

\section{A. Human walking trajectory model}

In [17], the authors designed an OC model which generates human-like trajectories between a starting and a goal position. This OC model is solved with a Differential Dynamic Programming (DDP) solver [28] from the Crocoddyl library [29].

${ }^{*}$ For sake of clarity we keep the term NMPC to differentiate the motion generation part from the prediction part. But in this paper, there is no state feedback, it is used as an optimal control approach. But the final aim is to perform NMPC. 
In this OC model, as in [15], the human is approximated by a holonomic system which follows the following dynamics :

$$
\left\{\begin{array}{l}
\dot{r}^{x}=\cos r^{\theta} \cdot v_{\text {forw }}-\sin r^{\theta} \cdot v_{\text {orth }} \\
\dot{r}^{y}=\sin r^{\theta} \cdot v_{\text {forw }}+\cos r^{\theta} \cdot v_{\text {orth }} \\
\dot{r}^{\theta}=\omega \\
\dot{v}_{\text {forw }}=u^{v_{f}} \\
\dot{v}_{\text {orth }}=u^{v_{o}} \\
\dot{\omega}=u^{\omega}
\end{array}\right.
$$

where $\left(r^{x}, r^{y}\right)$ is the horizontal position of the system and $r^{\theta}$ is its orientation with respect to a global frame. $v_{\text {forw }}$ and $v_{\text {orth }}$ are the forward and orthogonal velocities of the system with respect to a local frame.

Then, this OC model can be described as follows with $x=\left(r^{x}, r^{y}, r^{\theta}, v_{\text {forw }}, v_{\text {orth }}, \omega\right)^{T}$ and $u=\left(u^{v_{f}}, u^{v_{o}}, u^{\omega}\right)^{T}$ :

$$
\min _{x(.), u(.), T} \int_{0}^{T} \phi_{r}(x(t), u(t)) \mathrm{d} t+\phi_{t}(x(T))
$$

Under the following strict equality constraints : the dynamical constraint $\dot{x}=f(t, x(t), u(t))$ (Eq1) and the initial constraint $x(0)=x_{0}$.

In Eq 2, $T$ is the time needed to go from the starting position to the goal position and $\phi_{r}$ and $\phi_{t}$ are the running and terminal cost functions introduced in [17].

\section{B. Prediction model}

The previously described OC model was shown to generate paths which fit well human CoM trajectories in [17]. This is why, in this paper, a similar OC problem is addressed to predict human walking behaviour.

First, we hypothesise that a human is walking and that his CoM position in the horizontal plane and the posterioanterior orientation of his pelvis, denoted $\tilde{c}=\left(\tilde{c}^{x}, \tilde{c}^{y}, \tilde{c}^{\theta}\right)^{T}$, are recorded at all time with a sampling period $T_{O C}$. Then, the following assumptions are made:

- $\tilde{c}$ is piecewise constant on each interval $\left[k T_{O C},(k+\right.$ 1) $\left.T_{O C}\right]$ with $k \in \mathbb{N}$. Thus, at time $t=k T_{O C}$, the human trajectory can be described by $\left(\begin{array}{llll}\tilde{c}_{0} & \tilde{c}_{1} & \ldots & \tilde{c}_{k}\end{array}\right)^{T}$.

- The prediction process starts when $k \geq N_{0}-1$ as $N_{0}$ is the amount of measurements needed before being able to correctly predict the human future trajectory.

- The prediction process stops when the human stops walking at an a priori unknown time $k=n_{f}$.

- The prediction process is done on a sliding window of size $N_{O C}>N_{0}$.

- At time $t=k T_{O C}$, the prediction windows goes from $n+1$ to $n+N_{O C}$ and the prediction process uses the measured trajectory from $n+1$ to $n+N_{0}$ with $n \in \llbracket-1, n_{f}-N_{0}+1 \rrbracket$ defined as $n=k-N_{0}$.

Thus, at time $t=k T_{O C}$, the prediction process will compute $X_{n+1}=\left(\begin{array}{llllll}x_{n+1} & x_{n+2} & \ldots & x_{k} & \ldots & x_{n+N_{O C}}\end{array}\right)^{T}$. The optimal solution will be denoted $X_{n+1}^{*}$. This process is represented on Fig 3 .

To solve this prediction problem, an OC model has been developed based on the one described in Eq2 This model can be expressed as follows at time $t=k T_{O C}, k=n+N_{0}$ :

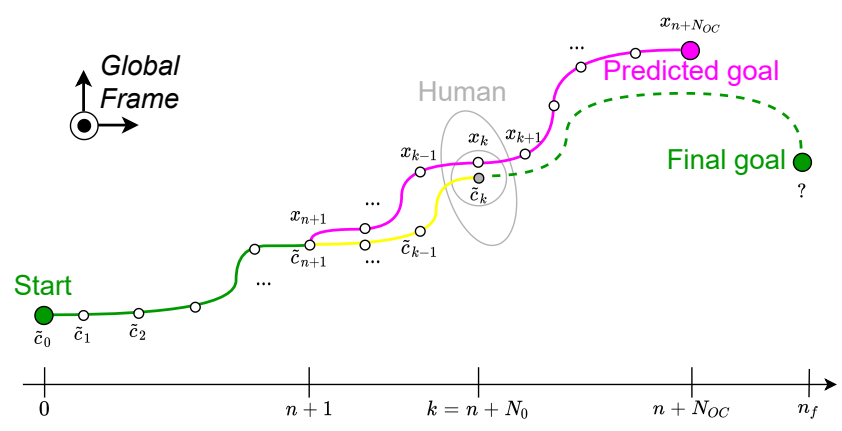

Fig. 3. Prediction problem representation at time $t=k T_{O C}, k=n+N_{0}$ (in green and yellow the whole past human trajectory, in stippled green the unknown future human trajectory and in purple the predicted trajectory)

$$
\left(X_{n+1}^{*}, U_{n+1}^{O C, *}\right)=\underset{X_{n+1}, U_{n+1}^{O C}}{\arg \min } \sum_{i=n+1}^{n+N_{O C}} \varphi_{i}\left(\tilde{c}_{i}, x_{i}, u_{i}\right)
$$

Under the following strict equality constraints:

$$
\begin{cases}\dot{x}=f(x(t), u(t)) & \text { Dynamical constraint }(\mathrm{Eq} 1) \\ x_{n+1}=\tilde{c}_{n+1} & \text { Initial constraints }\end{cases}
$$

With the following cost functions:

$$
\begin{gathered}
\forall i \in \llbracket n+1, k \rrbracket, \varphi_{i}\left(\tilde{c}_{i}, x_{i}, u_{i}\right)=\alpha_{0}+\alpha_{1} u_{i}^{v_{f} 2}+\alpha_{2} u_{i}^{v_{o} 2} \\
+\alpha_{3} u_{i}^{\omega 2}+\gamma_{0}\left(\left(\tilde{c}_{i}^{x}-r_{i}^{x}\right)^{2}+\left(\tilde{c}_{i}^{y}-r_{i}^{y}\right)^{2}\right)+\gamma_{1}\left(\tilde{c}_{i}^{\theta}-r_{i}^{\theta}\right)^{2} \\
\forall i \in \llbracket k+1, n+N_{O C} \rrbracket, \varphi_{i}\left(\tilde{c}_{i}, x_{i}, u_{i}\right)=\alpha_{0}+\alpha_{1} u_{i}^{v_{f} 2} \\
+\alpha_{2} u_{i}^{v_{o} 2}+\alpha_{3} u_{i}^{\omega 2}
\end{gathered}
$$

with $\left(\gamma_{0}, \gamma_{1}\right)=(10,10)$ heuristically chosen so that the beginning of the prediction fits as much as possible the measurements and $\left(\alpha_{0}, \alpha_{1}, \alpha_{2}, \alpha_{3}\right)=(7.9,4.0,20.1,1.0 \times$ $\left.10^{-6}\right)$ computed in [17].

Thus, at time $t=k T_{O C}, k=n+N_{0}$, this new OC model, also solved with a DDP solver from the Crocoddyl library, provides a predicted trajectory $X_{n+1}^{*}$.

\section{Prediction process simulation}

Thus, the described prediction process needs a human trajectory as an input. In this paper, we used trajectories of 10 healthy subjects walking from 40 starting positions to one goal position in front of a table collected as part of a study of human walking trajectories [16] [17].

To simulate a human walking, the recorded trajectory was sent at a given rate, simulating the human velocity, to the prediction process thanks to a ROS framework [30]. According to the chosen rate, the recorded trajectory could be sped up or slowed down. As part of this work, we hypothesise that a measured human trajectory is independent from its travel velocity. One simulation on the RViz software is shown on Fig 4 .

\section{WAlking Pattern Generator For Trajectory TRACKING}

Then, as the future trajectory of the human can now be computed thanks to the prediction model described in the previous section, only the robot gait remains to be generated 


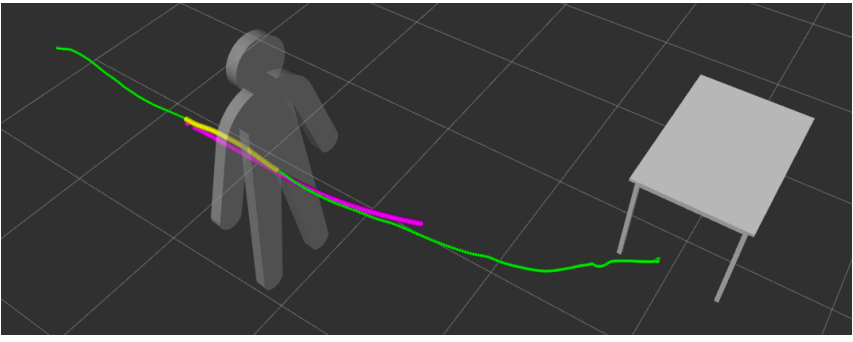

Fig. 4. Example of the current predicted trajectory (in purple) of a human trajectory (in green) from his latest past trajectory (in yellow) with $N_{0}=50$ and $N_{O C}=100$

to perform a proactive tracking of a human. In this part, a new WPG is designed to generate the CoM trajectory and the footsteps for a TALOS humanoid robot along the predicted trajectories. Our work is based on the NMPC developed in [26] which simultaneously solves the CoM and footsteps position and orientation problems. There are two main differences between the NMPC described in this paper and the one in [26]. Indeed, in this article :

- The controller is designed to track a reference trajectory rather than a reference velocity which implies a different cost function in comparison with [26].

- A terminal constraint on the Capture Point (CP) has been added to avoid the internal instability issue in the problem formulation.

In the following sections, a short description of the NMPC core equations is provided and more details can be found in [25] and [26].

\section{A. Description of the problem variables}

1) Center of Mass: As in [26], the robot CoM is controlled on a preview horizon of length $N_{W P G}$. On each sampling period of time $T_{W P G}$, the jerk is assumed to be piecewise constant i.e. $\forall t \in\left[k T_{W P G},(k+1) T_{W P G}\right]$ with $k \in$ $\llbracket 1, N_{W P G} \rrbracket, \dddot{c}_{k}^{\nu}(t)=\dddot{c}_{k}^{\nu}$ with $c$ the robot CoM position in the global frame and $\nu \in\{x, y\}$. Then, this leads to the following equation :

$$
\hat{c}_{k+j}^{\nu}=A^{j} \hat{c}_{k}^{\nu}+\sum_{i=0}^{j-1} A^{i} B \dddot{c}_{k+i}^{\nu}
$$

with $\hat{c}_{k}^{\nu}=\left(\begin{array}{c}c_{k}^{\nu} \\ \dot{c}_{k}^{\nu} \\ \ddot{c}_{k}^{\nu}\end{array}\right), A=\left(\begin{array}{ccc}1 & T_{W P G} & \frac{T_{W P G}^{2}}{2} \\ 0 & 1 & T_{W P G} \\ 0 & 0 & 1\end{array}\right)$ and $B=$ $\left(\begin{array}{c}\frac{T_{W P G}^{3}}{6} \\ \frac{T_{W P G}^{2}}{2} \\ T_{W P G}^{2}\end{array}\right)$. Thus, the CoM over the preview horizon and its derivatives are then defined as :

$$
\left\{\begin{array}{l}
C_{k+1}^{\nu}=\left(\begin{array}{lll}
c_{k+1}^{\nu} & \ldots & c_{k+N_{W P G}}^{\nu}
\end{array}\right)^{T}=S_{p} C_{k}^{\nu}+U_{p} \dddot{C}_{k}^{\nu} \\
\dot{C}_{k+1}^{\nu}=\left(\begin{array}{lll}
\dot{c}_{k+1}^{\nu} & \ldots & \dot{c}_{k+N_{W P G}}^{\nu}
\end{array}\right)^{T}=S_{v} C_{k}^{\nu}+U_{v} \dddot{C}_{k}^{\nu} \\
\ddot{C}_{k+1}^{\nu}=\left(\begin{array}{lll}
\ddot{c}_{k+1}^{\nu} & \ldots & \ddot{c}_{k+N_{W P G}}^{\nu}
\end{array}\right)^{T}=S_{a} C_{k}^{\nu}+U_{a} \dddot{C}_{k}^{\nu} \\
\dddot{C}_{k+1}^{\nu}=\left(\begin{array}{lll}
\dddot{c}_{k+1}^{\nu} & \ldots & \dddot{c}_{k+N_{W P G}}^{\nu}
\end{array}\right)^{T}
\end{array}\right.
$$

with $S_{p}, \quad S_{v}, \quad S_{a} \in \mathbb{R}^{N_{W P G} \times 3}$ and $U_{p}, \quad U_{v}$, $U_{a} \in \mathbb{R}^{N_{W P G} \times N_{W P G}}$ obtained from a recursive application of the CoM dynamics (Eq 6 .

2) Zero Moment Point: According to the LIPM, the ZMP can be expressed as a linear function of the CoM as follows:

$$
z_{k}^{\nu}=\left(\begin{array}{lll}
1 & 0 & -\frac{h}{g}
\end{array}\right) \hat{c}_{k}^{\nu}
$$

with $h$ the height of the CoM with respect to the ground and $g$ the gravity. Thus, the ZMP over the preview horizon can be expressed as:

$$
Z_{k+1}^{\nu}=\left(\begin{array}{lll}
z_{k+1}^{\nu} & \ldots & z_{k+N_{W P G}}^{\nu}
\end{array}\right)^{T}=S_{z} C_{k}^{\nu}+U_{z} \dddot{C}_{k}^{\nu}
$$

with $S_{z} \in \mathbb{R}^{N_{W P G} \times 3}$ and $U_{z} \in \mathbb{R}^{N_{W P G} \times N_{W P G} \text {. }}$

3) Footsteps: The position and orientation of the support foot are described by :

$$
F_{k+1}^{\eta}=\left(\begin{array}{lll}
f_{k+1}^{\eta} & \ldots & f_{k+N_{W P G}}^{\eta}
\end{array}\right)^{T}=v_{k+1} f_{k}^{\eta}+V_{k+1} \tilde{F}_{k+1}^{\eta}
$$

$f_{k}^{\eta}$ with $\eta=\{x, y, \theta\}$ is the current position and orientation of the support foot. $\tilde{F}_{k+1}^{\eta}$ of size $n_{D S}$ is the free variable of the problem with $n_{D S}$ the maximum number of double support phase in the preview. The vector $v_{k+1} \in \mathbb{R}^{N_{W P G}}$ and the matrix $V_{k+1} \in \mathbb{R}^{N_{W P G} \times n_{D S}}$ are called selection matrix, they indicate which foot is the support foot during the sampling interval. The footsteps placement strategy is defined in section III-B

Let $f_{k+1}^{\theta, R}$ and $f_{k+1}^{\theta, L}$ respectively be the orientation of the right foot and the left foot. The orientation of the free-flyer is defined as follows :

$$
\begin{gathered}
C_{k+1}^{\theta}=\frac{1}{2}\left(f_{k+1}^{\theta, R}+f_{k+1}^{\theta, L}\right), \dot{C}_{k+1}^{\theta}=\frac{1}{2}\left(\dot{f}_{k+1}^{\theta, R}+\dot{f}_{k+1}^{\theta, L}\right) \\
\text { and } \ddot{C}_{k+1}^{\theta}=\frac{1}{2}\left(\ddot{f}_{k+1}^{\theta, R}+\ddot{f}_{k+1}^{\theta, L}\right)
\end{gathered}
$$

4) Capture Point: The CP was introduced by [31] and [32]. It can be derived from the LIPM system equations and is defined as follows:

$$
\xi_{k}^{\nu}=c_{k}^{\nu}+\frac{\dot{c}_{k}^{\nu}}{\omega}
$$

with $\omega=\sqrt{\frac{h}{g}}$. Thus, the CP over the preview horizon can be defined as follows :

$$
\Xi_{k+1}^{\nu}=\left(\begin{array}{lll}
\xi_{k+1}^{\nu} & \ldots & \xi_{k+N_{W P G}}^{\nu}
\end{array}\right)^{T}=S_{\xi} C_{k}^{\nu}+U_{\xi} \dddot{C}_{k}^{\nu}
$$

with $S_{\xi}=S_{p}+\frac{1}{\omega} S_{v}$ and $U_{\xi}=U_{p}+\frac{1}{\omega} U_{v}$. In section III-C a terminal constraint on the CP is defined.

\section{B. Cost function for trajectory tracking}

As a reminder, in this work, we want the humanoid robot to follow the predicted trajectory defined in section II-B Moreover, as we want the robot to dynamically follow a human, the robot needs to follow the trajectory with the same velocity as the human walking velocity. In this part, the cost function of this NMPC, which is one of the contributions of this paper, is described.

At time $t=k T_{O C}, k=n+N_{0}$, the following data, defined in section II-B, can be given to the controller: 


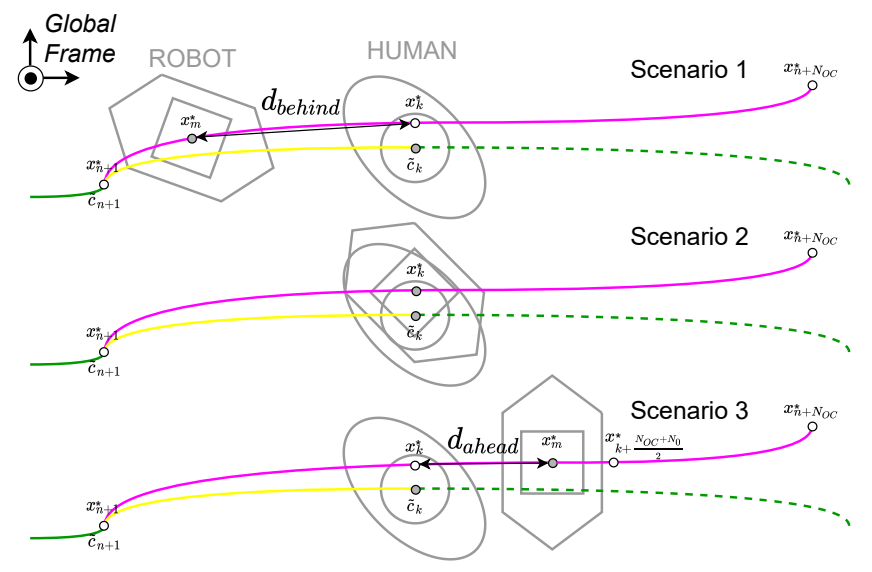

Fig. 5. Representation of the 3 possible scenarios : at the top, the robot walks behind the human, on the middle they are synchronized, at the bottom the robot walks ahead of the human

- The measured human trajectory until time $t$ : $\left(\begin{array}{llll}\tilde{c}_{0} & \tilde{c}_{1} & \ldots & \tilde{c}_{k}\end{array}\right)^{T}$

- The predicted trajectory at time $t: X_{n+1}^{*}=$ $\left(\begin{array}{lllll}x_{n+1}^{*} & \ldots & x_{k}^{*} & \ldots & x_{n+N_{O C}}^{*}\end{array}\right)^{T}$.

From the measured human trajectory, an average current human walking velocity can be computed :

$$
\bar{v}_{n+1}=\frac{\sqrt{\left(\tilde{c}_{k}^{x}-\tilde{c}_{n+1}^{x}\right)^{2}+\left(\tilde{c}_{k}^{y}-\tilde{c}_{n+1}^{y}\right)^{2}}}{N_{0} \times T_{O C}}
$$

At this point, three scenarios should be considered :

1) The robot walks behind the human at a constant distance $d_{\text {behind }}$.

2) The robot and the human act synchronously so that no distance divides them. Experimentally, this scenario can be achieved by putting the robot and its human partner side by side to show that the robot is mimicking the human motions without any delay.

3) The robot walks ahead of the human at a constant distance $d_{\text {ahead. }}$

In all those scenarios, the robot is trying to follow the same trajectory as the human. According to which scenario is played, the part of the predicted trajectory used in the cost function will be different. Indeed, it will respectively be $X_{n+1}^{*(m)}=\left(\begin{array}{lll}x_{m}^{*} & \ldots & x_{n+N_{O C}}^{*}\end{array}\right)^{T}$ with :

4) $0 \leq m<k$ such that $\sqrt{\left(c_{k}^{x}-c_{m}^{x}\right)^{2}+\left(c_{k}^{y}-c_{m}^{y}\right)^{2}} \approx$ $d_{\text {behind }}$

5) $c_{m}^{\nu}=c_{k}^{\nu}$

6) $k<m \leq k+\left\lfloor\frac{N_{O C}-N_{0}}{2}\right\rfloor$ such that $\sqrt{\left(c_{k}^{x}-c_{m}^{x}\right)^{2}+\left(c_{k}^{y}-c_{m}^{y}\right)^{2}} \approx d_{\text {ahead }}$

Those scenarios are represented on Fig 5

Then, to respect the human walking velocity, not the entirety of $X_{n+1}^{*(m)}$ has to be sent to the NMPC. Indeed, the robot should travel during $T_{W P G} \times N_{W P G}$ the same distance as the human, namely $\bar{v}_{n+1} \times T_{W P G} \times N_{W P G}$. So, we should compute the index $l \in \llbracket m, n+N_{O C} \rrbracket$ such as $\sum_{i=m}^{l} \sqrt{\left(r_{i}^{x, *}-r_{i-1}^{x, *}\right)^{2}+\left(r_{i}^{y, *}-r_{i-1}^{y, *}\right)^{2}} \approx \bar{v}_{n+1} \times T_{W P G} \times$ $N_{W P G}$. Then $\left(\begin{array}{lll}x_{m}^{*} & \ldots & x_{l}^{*}\end{array}\right)^{T}$ can be interpolated in order to count $N_{W P G}$ terms. Then, the following vector can be sent to the NMPC :

$$
C_{k+1}^{\eta, r e f}=\left(\begin{array}{lll}
\tilde{x}_{m}^{*} & \ldots & \tilde{x}_{l}^{*}
\end{array}\right)^{T}
$$

Now, the cost function used in this new NMPC can be defined as follows :

$J\left(U_{k}^{W P G}\right)=\frac{\alpha}{2} J_{1}\left(U_{k}^{W P G}\right)+\frac{\beta}{2} J_{2}\left(U_{k}^{W P G}\right)+\frac{\gamma}{2} J_{3}\left(U_{k}^{W P G}\right)$

with $U_{k}^{W P G}=\left(\begin{array}{lllll}\dddot{C}_{k}^{x} & \tilde{F}_{k}^{x} & \dddot{C}_{k}^{y} & \tilde{F}_{k}^{y} & \tilde{F}_{k}^{\theta}\end{array}\right)^{T}$ and $\alpha, \beta$ and $\gamma$ the same cost weights as in [26].

$J_{1}$ is the cost function ensuring the tracking of the predicted trajectory and is defined as follows :

$$
\begin{array}{r}
J_{1}\left(U_{k}^{W P G}\right)=\left\|C_{k+1}^{x}-C_{k+1}^{x, r e f}\right\|_{2}^{2}+\left\|C_{k+1}^{y}-C_{k+1}^{y, r e f}\right\|_{2}^{2} \\
+\left\|C_{k+1}^{\theta}-C_{k+1}^{\theta, r e f}\right\|_{2}^{2}
\end{array}
$$

$J_{2}$ is the cost function placing the ZMP as close as possible to the projection of the ankle on the ground, this cost defined the footsteps placement strategy:

$$
J_{2}\left(U_{k}^{W P G}\right)=\left\|F_{k+1}^{x}-Z_{k+1}^{x}\right\|_{2}^{2}+\left\|F_{k+1}^{y}-Z_{k+1}^{y}\right\|_{2}^{2}
$$

$J_{3}$ is the cost function minimizing the jerk :

$$
J_{3}\left(U_{k}^{W P G}\right)=\left\|\dddot{C}_{k+1}^{x}\right\|_{2}^{2}+\left\|\dddot{C}_{k+1}^{y}\right\|_{2}^{2}
$$

Thus, the problem solved in this NMPC is the following:

$$
\min _{U_{k}^{W P G}} J\left(U_{k}^{W P G}\right)
$$

under the constraints presented in the following section.

\section{Constraints of the NMPC}

Among the constraints presented in [26], the balance, the footstep feasibility and the foot orientation have been kept to perform the trajectory tracking with the NMPC defined in the previous section.

Moreover, one more constraint has been added to deal with the inherent instability of the LIPM system equations. Indeed, in the LIPM-based models, the CoM can diverge exponentially even if the ZMP is maintained in the support polygon. Thus, this generates an unfeasible walking pattern [33]. One way to solve this instability is to add a terminal constraint [24]. This terminal constraint can be a capturability constraint which imposes the robot to stop at the end of the planning horizon [34].

Thus, in this paper, a terminal inequality constraint which imposes the CP to be in the support polygon at the end of the preview horizon was added. This terminal constraint can be expressed as :

$n_{i, n+N_{W P G}}^{\nu} \xi_{n+N_{W P G}}^{\nu}\left(U_{n+N_{W P G}}^{W P G}\right)<n_{i, n+N_{W P G}}^{\nu} V_{i, n+N_{W P G}}^{\nu}$

$\forall i \in \llbracket 1,4 \rrbracket, n_{i}=\left(\begin{array}{l}V_{i+1}^{y}-V_{i}^{y} \\ V_{i}^{x}-V_{i+1}^{x}\end{array}\right)$ is the vector orthogonal to the edges $V_{i} V_{i+1}$ of the support foot and $V_{i}$ its vertices. 


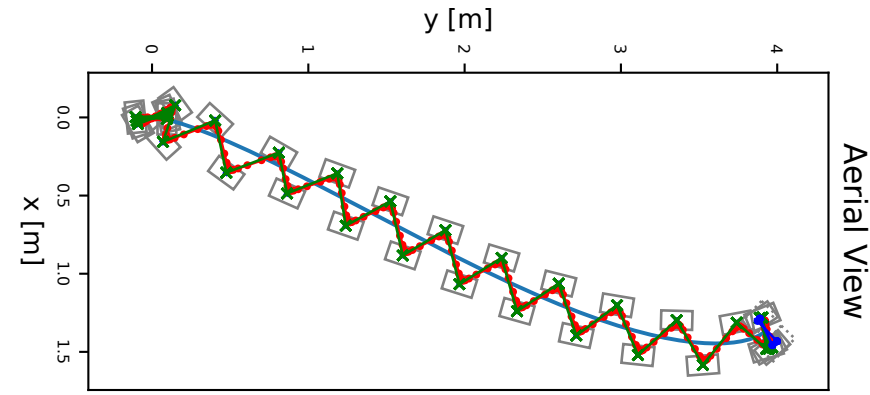

Fig. 6. Example of a CoM trajectory (in red) and the footsteps generated (in green) with the presented WPG along a given trajectory generated with the OC model presented in section II-A (in blue)

\section{Solver}

As in [26], this optimisation problem is turned into a least squares problem using a generalized Gauss-Newton method. Thus, solving the previously described problem amounts to solving a Sequential Quadratic Problem (SQP). This SQP is solved with the qpOASES library [35]. More details about this transformation can be found in [26].

\section{E. Generation of robot CoM trajectory and footsteps}

To test the NMPC behaviour, trajectories generated with the OC model presented in section [II-A were given to the NMPC with a constant velocity $\bar{v}_{n+1}=0.25 \mathrm{~m} / \mathrm{s}$. Moreover, for steep changes in the orientations, a threshold on the velocity was defined to prevent the constraints to become unreachable. The result obtained for a trajectory with $\left(x_{0}, y_{0}, \theta_{0}\right)=(0,0,0)$ as a starting pose and $\left(x_{f}, y_{f}, \theta_{f}\right)=$ $(1.35,4, \pi)$ as a goal pose is represented on Fig 6. This version of the WPG is called offline WPG. An online version coupled to the prediction process is described in the following section. Let us note that the computational time for each iteration of the NMPC is about $2 \times 10^{-3} \mathrm{~s}$ with $N_{W P G}=16$ and $T_{W P G}=0.2 \mathrm{~s}$

\section{Simulation ON A TALOS Robot}

\section{A. Coupling of the Prediction Process and the WPG}

As a reminder, the purpose of this paper is to design a whole architecture that allows a humanoid robot to smoothly follow a human who walks to an a priori unknown goal while predicting his future trajectory. This tracking is aimed to be proactive as a prediction process estimates the human future trajectory so that the future steps of the robot planned by the WPG are already in the right direction. This may cancel the reaction time of the robot and make the tracking smoother. The coupling of the prediction process described in Sec II and the WPG for a TALOS humanoid robot described in $\mathrm{Sec}$ III is a first step towards this purpose.

This coupling can be described as follows. First, a prerecorded human trajectory is streamed and retrieved by the prediction process like in SedII-C Then, it sends the predicted trajectory to the WPG which computes, thanks to a NMPC, the robot CoM and feet positions and orientations over the preview horizon with respect to the human velocity computed with $\mathrm{Eq} 13$ All those data are shared through a ROS framework and displayed on the RViz software
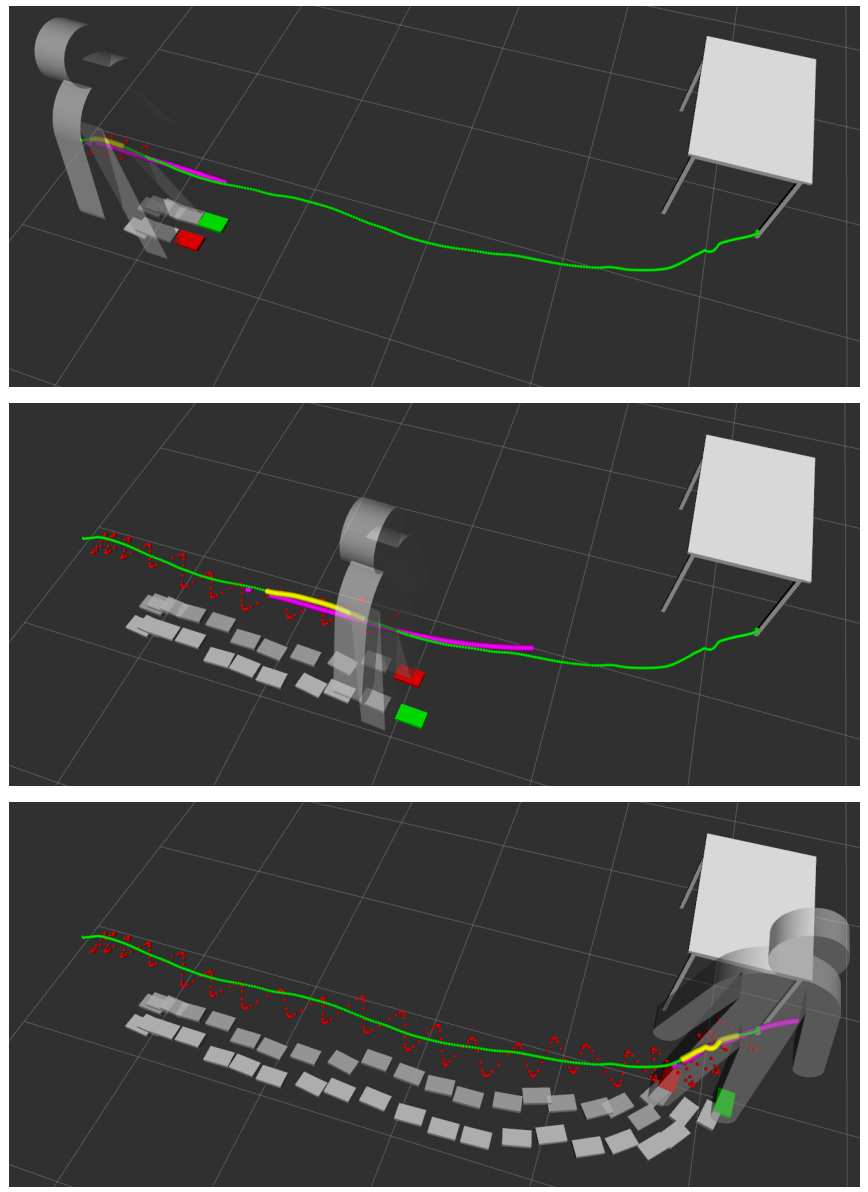

Fig. 7. The robot CoM (in red) and footsteps (past steps in grey, current support foot in red and future support foot in green) are generated from the current predicted human trajectory (in purple) with $N_{0}=50$ and $N_{O C}=$ 100

as shown on Fig7 for a trajectory with $\left(x_{0}, y_{0}, \theta_{0}\right)=$ $(-1.5,4,0)$ as a starting pose and $\left(x_{f}, y_{f}, \theta_{f}\right)=\left(0,0, \frac{\pi}{2}\right)$. In this figure, the chosen scenario is the second one on Fig 5 namely the synchronization of a robot and a human. This coupling matches with the blue boxes represented in Fig 2 which shows the whole framework. In the next section, the last steps of this process, namely the whole-body controller (the green box on Fig 2) and the simulation on the TALOS robot on Gazebo are presented.

All the results and simulations resulting of this coupling are reproducible as all the libraries are opensource and the source code and the data are available on: https://github.com/imaroger/human_ walking_trajectory_prediction

\section{B. Whole-body controller}

The whole-body controller computes a stable command from the reference trajectories and the actual state of the robot. In this paper, the controller used in simulation is a Weighted Quadratic Program (WQP), which solves the inverse dynamics of a robot in rigid contact with the environment [36]. It has been successfully tested on the humanoid robot TALOS in simulations in [37]. The controller takes as inputs the reference trajectories of the WPG and implements 

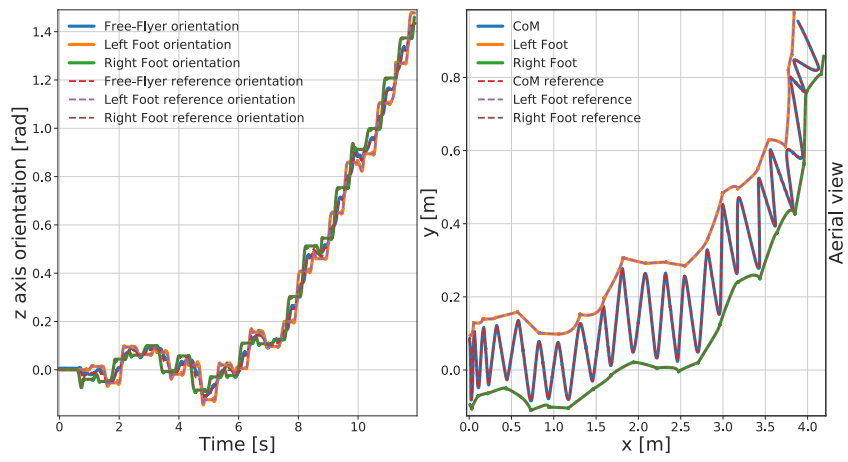

Fig. 8. Tracking of the CoM and Feet trajectories in the Gazebo simulation.

task functions with them, as acceleration-based tracking law. The controller optimises a cost function using these tasks, prioritised with weights, while respecting constraints such as the robot dynamics and feet contacts. The interest of this controller is that it implements an Angular Momentum (AM) regularisation task, which allows to control the angular momentum part generated by the contact transition [38]. In [37] three controllers are compared, in this paper, the torque controller is used.

\section{Simulation}

The simulation has been realized using Gazebo on a standard laptop (Intel CPU i7-8850H @ 2.6 GHz). The reference trajectories from the WPG are registered in files which are read by the whole-body controller during the simulation. The controller computes the desired torque for all the joints of the robot at $1 \mathrm{kHz}$ and sends this command to the simulated robot in Gazebo. The simulation result of the same trajectory presented in Fig 7 is shown in Fig 1 . The CoM and feet references are well followed by the controller, their tracking are presented in Fig 8 , allowing the robot to successfully perform the motion. Compared to [37], gains tuning were needed to make the controller successfully perform the trajectory wide side steps (the CoM proportional gains were raised to 800 and the AM ones decreased to 2.5). The whole prediction process and some simulations are shown in the video available at https: //youtu.be/hu-cuUYl-58

\section{DISCUSSION}

In this paper, a whole framework allowing a proactive co-navigation task is presented. This framework including a prediction of human trajectory and a WPG generating the CoM and the footsteps of a TALOS humanoid robot along the predicted trajectory gave good results in simulations as it is presented in the previous section.

Human walking trajectory model. The presented OC model used to predict the human trajectory depends a lot on the chosen human trajectory model. In this work, the chosen model is the one described in section II-A. This model has been optimised in [17] to generate smooth trajectory of the human CoM. That is to say that it does not take into account the oscillations of the CoM due to the steps.
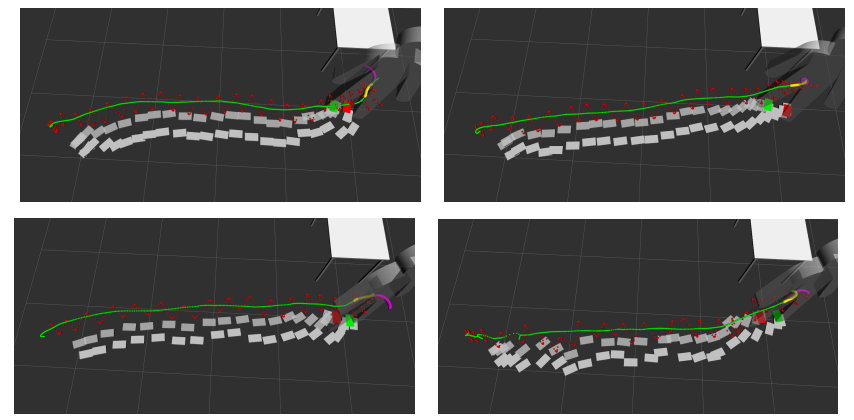

Fig. 9. Result of the coupling of the prediction model and the WPG for 4 different human subjects

So, another model of human gait taking the steps into account could be consider in case of the prediction model needs to be more precise. Moreover, the chosen human trajectory model has been shown to fit well the average human behaviour while its performance could be poorer when apply to individual subjects. However, when testing the prediction model over trajectories of numerous subjects, it seems to achieve its goals whoever the subject is. The results of 4 predictions for 4 different subjects going from the same starting pose $\left(x_{0}, y_{0}, \theta_{0}\right)=\left(-1.5,4, \frac{\pi}{2}\right)$ to the same goal pose $\left(x_{f}, y_{f}, \theta_{f}\right)=\left(0,0, \frac{\pi}{2}\right)$ are presented on Fig 9 This demonstrates that the coupling of the prediction process and the WPG provides consistent results which do not depend on the human partner with whom the robot interacts.

Role of the parameters in the whole process performance. On the one hand, the prediction process efficiency depends a lot on the parameters $N_{0}$ and $N_{O C}$. Indeed, when performing multiple tests, it seems that the greater $N_{0}$ is and the closer $N_{O C}$ is from $N_{0}$ the more precise the prediction will be. However, to give a satisfying result, the prediction process accepts a wide range of parameters. When taking $N_{0}=15$ and $N_{O C}=75$ the process is still able to predict the human behaviour even if this prediction is less accurate than with $N_{0}=50$ for example. Yet, for the whole-body controller using $N_{O C}=100$ leads to a more stable motion. A numerical assessment of the prediction model is presented in [39]. On the other hand, the precision of the human tracking performed by the WPG relies on the chosen scenario and on the potential chosen distance to the human. The greater $d_{\text {behind }}$ is, the cleaner the footsteps are because the part of the predicted trajectory used by the WPG is mostly in the the measured part. On the opposite, the greater $d_{\text {ahead }}$ is, the messier the footsteps are because all those generated footsteps are based on the prediction which can vary a lot according to the measurements.

Feasibility of the $S Q P$ for high velocity When the robot is required to walk at velocity higher than $0.25 \mathrm{~m} / \mathrm{s}$ following a trajectory with a non-zero curvature, the SQP turns out to be infeasible and the robot cannot walk with its human partner anymore. This may be solved relaxing the constraint on the foot rotation or adapting the foot constraint bounds 
for a TALOS robot as, in this paper, we used the ones computed for HRP-2 in [26]. We could also use another solver like the ones implemented in the Crocoddyl library [29]. The improvement of the WPG to achieve a higher maximum velocity in non-zero curvature trajectories will be the focus of future works.

Toward a full online application on the real robot. The test on the TALOS robot is an ongoing work. Torque-based walking has been successfully realized. However this walking pattern generator has some limited speed. Developments are under way to reach a sufficient speed to realize a humanhumanoid robot interaction.

\section{CONCLUSION}

In this paper, we aim to ease the tracking of a human by a humanoid robot by helping the robot to predict, and thus anticipate, its partner behaviour. To this end, we first present a prediction model of human walking trajectory which generates the future trajectory of a human from its recent past trajectory. Then, a WPG is designed to generate a TALOS humanoid robot CoM and footsteps along the predicted trajectory in real time. Finally the CoM and footsteps trajectories resulting from the WPG are given to a torque whole-body controller which computes a stable command for the robot to follow. This whole framework has been successfully tested in real-time simulation. Thus, in this work, we achieve to develop a whole framework to control a simulated TALOS humanoid robot in order to make him proactively walk along with a human partner.

\section{REFERENCES}

[1] N. Jarrassé, V. Sanguineti, and E. Burdet, "Slaves no longer: review on role assignment for human-robot joint motor action," Adaptive Behavior, SAGE Publications, 2013.

[2] A. Bussy, P. Gergondet, A. Kheddar, F. Keith, and A. Crosnier, "Proactive behavior of a humanoid robot in a haptic transportation task with a human partner," in IEEE RO-MAN, 2012.

[3] K. Otani, K. Bouyarmane, and S. Ivaldi, "Generating assistive humanoid motions for co-manipulation tasks with a multi-robot quadratic program controller," in IEEE ICRA, 2018.

[4] P. Teja S. and R. Alami, "Hateb-2: Reactive planning and decision making in human-robot co-navigation," in IEEE RO-MAN, 2020.

[5] J. Lanini, A. Duburcq, H. Razavi, C. Le Goff, and A. Ijspeert, "Interactive locomotion: Investigation and modeling of physicallypaired humans while walking," PLOS ONE, 2017.

[6] J. Lanini, H. Razavi, J. Urain, and A. Ijspeert, "Human intention detection as a multiclass classification problem: Application in physical human-robot interaction while walking," IEEE RA-L, 2018.

[7] D. Aarno and D. Kragic, "Motion intention recognition in robot assisted applications," Robotics and Autonomous Systems, 2008.

[8] R. Kelley, M. Nicolescu, A. Tavakkoli, M. Nicolescu, C. King, and G. Bebis, "Understanding human intentions via hidden markov models in autonomous mobile robots," in ACM/IEEE HRI, 2008.

[9] Q. Li, Z. Zhang, Y. You, Y. Mu, and C. Feng, "Data driven models for human motion prediction in human-robot collaboration," IEEE Access, 2020.

[10] M. Awais and D. Henrich, "Human-robot collaboration by intention recognition using probabilistic state machines," in $R A A D, 2010$.

[11] D. A. Winter, "Biomechanics and motor control of human gait," in Waterloo, Ont. : University of Waterloo Press, 1991

[12] M. Raković, S. Savić, J. Santos-Victor, M. Nikolić, and B. Borovac, "Human-inspired online path planning and biped walking realization in unknown environment," Frontiers in Neurorobotics, 2019.
[13] A. Papadopoulos, L. Bascetta, and G. Ferretti, "Generation of human walking paths," in IEEE IROS, 2013.

[14] G. Arechavaleta, J.-P. Laumond, H. Hicheur, and A. Berthoz, "On the nonholonomic nature of human locomotion," Autonomous Robots, 2008.

[15] K. Mombaur and J.-P. Laumond, "From human to humanoid locomotion - an inverse optimal control approach," Autonomous Robots, 2010.

[16] I. Maroger, O. Stasse, and B. Watier, "Walking human trajectory models and their application to humanoid robot locomotion," in IEEE/RSJ IROS, 2020.

[17] I. Maroger, O. Stasse, and B. Watier, "Inverse optimal control to model human trajectories during gait," 2021, unpublished.

[18] S. Kajita, O. Matsumoto, and M. Saigo, "Real-time 3d walking pattern generation for a biped robot with telescopic legs," in IEEE ICRA, 2001.

[19] P. Wieber, "On the stability of walking systems," in Proceedings of the International Workshop on Humanoid and Human Friendly Robotics, 2002

[20] S. Kajita, F. Kanehiro, K. Kaneko, K. Fujiwara, K. Harada, K. Yokoi, and H. Hirukawa, "Biped walking pattern generation by using preview control of zero-moment point," in IEEE ICRA, 2003.

[21] P. Wieber, "Trajectory free linear model predictive control for stable walking in the presence of strong perturbations," in IEEE-RAS $\mathrm{Hu}$ manoids, 2006.

[22] S. Faraji, S. Pouya, C. G. Atkeson, and A. J. Ijspeert, "Versatile and robust $3 \mathrm{~d}$ walking with a simulated humanoid robot (atlas): A model predictive control approach," in IEEE ICRA, 2014.

[23] R. J. Griffin and A. Leonessa, "Model predictive control for dynamic footstep adjustment using the divergent component of motion," in IEEE ICRA, 2016.

[24] N. Scianca, D. De Simone, L. Lanari, and G. Oriolo, "Mpc for humanoid gait generation: Stability and feasibility," IEEE T-RO, 2020

[25] A. Herdt, N. Perrin, and P. Wieber, "Walking without thinking about it," in IEEE/RSJ IROS, 2010.

[26] M. Naveau, M. Kudruss, O. Stasse, C. Kirches, K. Mombaur, and P. Souères, "A reactive walking pattern generator based on nonlinear model predictive control," IEEE RA-L, 2017.

[27] S. Caron and A. Kheddar, "Dynamic walking over rough terrains by nonlinear predictive control of the floating-base inverted pendulum,' in IEEE/RSJ IROS, 2017.

[28] Y. Tassa, N. Mansard, and E. Todorov, "Control-limited differential dynamic programming," in IEEE ICRA, 2014.

[29] C. Mastalli, R. Budhiraja, W. Merkt, G. Saurel, B. Hammoud, M. Naveau, et al., "Crocoddyl: An efficient and versatile framework for multi-contact optimal control," in IEEE ICRA, 2020.

[30] Stanford Artificial Intelligence Laboratory et al., "Robotic operating system.” [Online]. Available: https://www.ros.org

[31] J. Pratt, J. Carff, S. Drakunov, and A. Goswami, "Capture point: A step toward humanoid push recovery," in IEEE-RAS Humanoids, 2006.

[32] A. Hof, M. Gazendam, and W. Sinke, "The condition for dynamic stability," Journal of Biomechanics, 2005.

[33] J. Englsberger, C. Ott, M. A. Roa, A. Albu-Schäffer, and G. Hirzinger, "Bipedal walking control based on capture point dynamics," in IEEE/RSJ IROS, 2011.

[34] T. Koolen, T. De Boer, J. Rebula, A. Goswami, and J. Pratt, "Capturability-based analysis and control of legged locomotion, part 1: Theory and application to three simple gait models," The International Journal of Robotics Research, 2012.

[35] H. Ferreau, C. Kirches, A. Potschka, H. Bock, and M. Diehl, "qpOASES: A parametric active-set algorithm for quadratic programming," Mathematical Programming Computation, 2014.

[36] A. Herzog, N. Rotella, S. Mason, F. Grimminger, S. Schaal, and L. Righetti, "Momentum control with hierarchical inverse dynamics on a torque-controlled humanoid," Autonomous Robots, 2014.

[37] N. Ramuzat, G. Buondonno, S. Boria, and O. Stasse, "Comparison of position and torque whole body control schemes on the talos humanoid robot," 2021, unpublished.

[38] S. Kajita, F. Kanehiro, K. Kaneko, K. Fujiwara, K. Harada, K. Yokoi, and H. Hirukawa, "Resolved momentum control: Humanoid motion planning based on the linear and angular momentum," in IEEE/RSJ IROS, 2003.

[39] I. Maroger, O. Stasse, and B. Watier, "Metrics to assess a human trajectory prediction model during gait," 2021, unpublished. 